\title{
Systems Biology of the Self-regulating Morphogenetic Gradient of the Xenopus Gastrula
}

\author{
Jean-Louis Plouhinec and E. M. De Robertis
}

Howard Hughes Medical Institute and Department of Biological Chemistry, University of California, Los Angeles, California 90095-1662

Correspondence: ederobertis@mednet.ucla.edu

The morphogenetic field concept was proposed by experimental embryologists to account for the self-regulative behavior of embryos. Such fields have remained an abstract concept until the recent identification of their molecular components using a combination of genetics, biochemistry, and theoretical modeling. One of the best studied models of a morphogenetic field is the Dorsal-Ventral (D-V) patterning of the early frog embryo. This patterning system is regulated by the bone morphogenetic protein (BMP) signaling pathway and an intricate network of secreted protein antagonists. This biochemical pathway of interacting proteins functions in the extracellular space to generate a D-V gradient of BMP signaling, which is maintained during extensive morphogenetic movements of cell layers during gastrulation. The D-V field is divided into a dorsal and a ventral center, in regions of low and high BMP signaling respectively, under opposite transcriptional control by BMPs. The robustness of the patterning is assured at two different levels. First, in the extracellular space by secreted BMP antagonists that generate a directional flow of BMP ligands to the ventral side. The flow is driven by the regulated proteolysis of the Chordin inhibitor and by the presence of a molecular sink on the ventral side that concentrates BMP signals. The tolloid metalloproteinases and the Chordin-binding protein Crossveinless-2 (CV2) are key components of this ventral sink. Second, by transcriptional feedback at the cellular level: The dorsal and ventral signaling centers adjust their size and level of BMP signaling by transcriptional feedback. This allows cells on one side of a gastrula containing about 10,000 cells to communicate with cells in the opposite pole of the embryo.

unique aspect of embryology is its vocation A to understand the ability of biological systems to generate self-regulating patterns after experimental perturbation. This ability is crucial to ensure the robustness of embryonic development, and is reused in the adult for the homeostasis and regeneration of tissues and organs. This property was discovered by early experimental embryologists who coined the term "morphogenetic field" to describe groups of cells that can differentiate autonomously and regenerate missing parts (for review, see De Robertis et al. 1991; Eivers et al. 2008).

An important step in understanding the behavior of morphogenetic fields has been the concept of diffusing chemical signals or

Editors: James Briscoe, Peter Lawrence, and Jean-Paul Vincent

Additional Perspectives on Generation and Interpretation of Morphogen Gradients available at www.cshperspectives.org

Copyright (C) 2009 Cold Spring Harbor Laboratory Press; all rights reserved; doi: 10.1101/cshperspect.a001701

Cite this article as Cold Spring Harb Perspect Biol 2009;1:a001701 
“morphogens" (Turing 1952). Wolpert (1969) proposed in his "French flag" model that a diffusing morphogen, flowing over a field of cells from a source to a sink, can form a linear gradient and pattern this field, provided that the cells are able to respond to different concentrations of the morphogen. Crick (1970) showed via a back of the envelope calculation that this model was physically plausible because the reaching of a steady-state gradient could occur in a reasonable amount of time in a field of $1 \mathrm{~mm}$ size. Turing (1952) was the first to formulate reaction-diffusion equations for a morphogen diffusing in a field of cells. Gierer and Meinhardt (1972) further showed that an activator and an inhibitor pair diffusing from the same source can generate stable patterns, provided the inhibitor diffuses faster than the activator. These simple models have proven to be very useful to qualitatively interpret embryological observations such as the patterning of the early Drosophila blastoderm by bicoid (Driever and Nüsslein-Volhard 1988; Gregor et al. 2007) or of the Drosophila segment by hedgehog (Heemskerk and DiNardo 1994).

\section{THE D-V GASTRULA MORPHOGENETIC FIELD}

One of the best studied self-regulating fields is the amphibian gastrula. The $\mathrm{D}-\mathrm{V}$ patterning system is responsible for the histotypic differentiation of the embryo, in the ectoderm instructing the choice between epidermis and neural tissue, and in the mesoderm between notochord, somite, kidney, lateral plate, and blood island differentiation. Spemann and Mangold (1924) discovered that the patterning of the gastrula D-V field was directed by an organizing center, the dorsal lip of the blastopore, which could induce a secondary axis with normal D-V and Antero-Posterior (A-P) patterning when transplanted into a host embryo. Soon, equivalent inducing tissues were found in other vertebrate embryos (Waddington 1932), and even in spiders in which a structure called the cumulus can trigger the generation of a second axis when transplanted (Holm 1952; Oda and Akiyama-Oda 2008).
An important recent advance was the realization that the frog dorsal organizer is mirrored by a ventral center, and that the gastrula $\mathrm{D}-\mathrm{V}$ pattern emerges from the balanced activities of these two centers (De Robertis and Kuroda 2004). The ventral center went unnoticed for many years because at the start of gastrulation it occupies a broad region that only becomes restricted to the ventral side by mid gastrula. As shown in Figure 1, the ventral center, as well as the dorsal center, displays inducing activity over great distances after transplantation into BMP-depleted embryos (Reversade and De Robertis 2005).

The advent of molecular genetics allowed the discovery of the molecular components regulating the D-V axis. Genetic screens conducted either in Drosophila (Rusch and Levine 1996) or zebrafish (Schier and Talbot 2005; Kondo 2007) identified mutations in genes mostly belonging to the bone morphogenetic protein (BMP) pathway. Indeed, in both vertebrates and arthropods, a gradient of BMP signaling controls $\mathrm{D}-\mathrm{V}$ patterning, although the gradient was inverted during evolution (De Robertis 2008). This gradient can be visualized in the Xenopus gastrula, as shown in Figure 2, through the carboxy-terminal phosphorylation of Smad1/5/8, the transcription factor that is phosphorylated by BMP receptors. The D-V patterning system has revealed an astonishing molecular conservation of the patterning molecular mechanisms between vertebrates and arthropods, and therefore was an ancestral feature of all bilateria (Holley et al. 1995; De Robertis and Sasai 1996; De Robertis 2008). From a practical point of view, these conserved features have led to rapid progress in the understanding of D-V patterning, through the full use of the diversity of approaches available in different model organisms.

\section{MOLECULAR ANATOMY OF THE GASTRULA FIELD}

Molecular cloning of the Xenopus patterning components has uncovered many proteins controlling $\mathrm{D}-\mathrm{V}$ patterning, as summarized in the biochemical pathway shown in Figure 3. D-V 
Systems Biology of the Morphogenetic Gradient of the Xenopus Gastrula
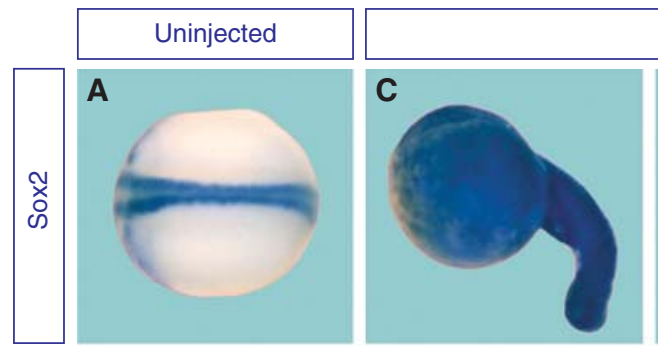

Admp/Bmp2/4/7 MOs
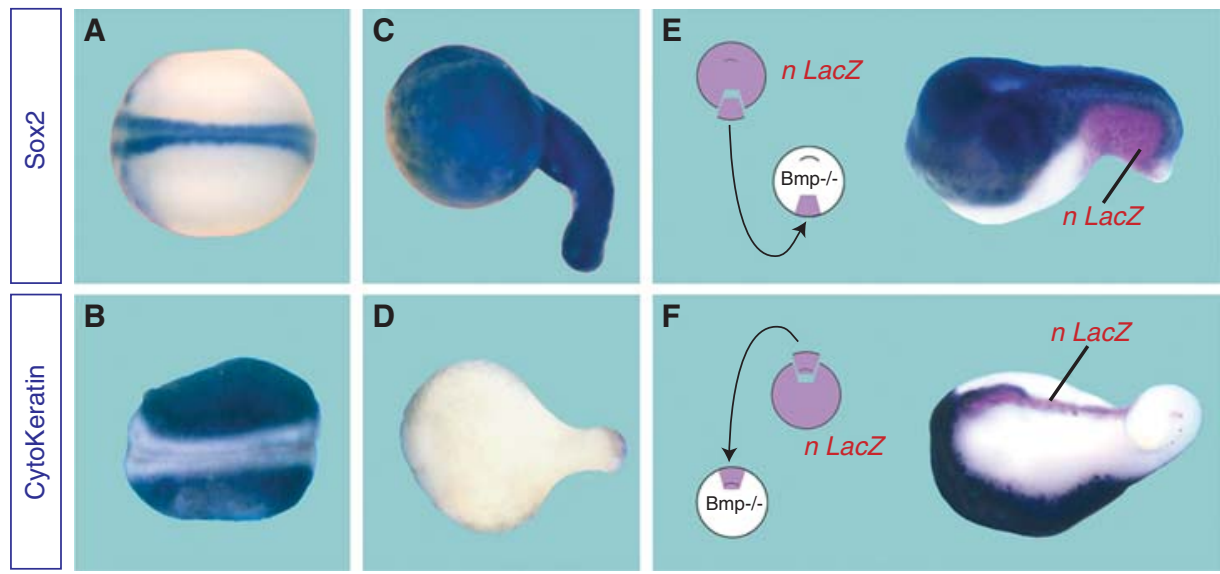

Figure 1. The dorsal and ventral gastrula centers have signaling activity. Xenopus embryos were injected with a cocktail of morpholinos (MOs) to deplete four BMPs (BMP2/4/7 and ADMP), and stained for a central nervous system (CNS) marker (Sox2 mRNA, top row) or an epidermal marker (Cytokeratin mRNA, bottom row). ( $A, B)$ an uninjected embryo displays a normal CNS and epidermis. $(B, D)$ BMP depletion converts the whole ectoderm into nervous tissue and cytokeratin staining is lost. $(E, F)$ The graft of a ventral or dorsal center (marked with nuclear LacZ lineage tracer) restores dorsal-ventral (D-V) patterning, even at a considerable distance from the graft. These experiments show first that BMP knockdown causes ubiquitous neural induction and second that the dorsal and ventral centers are both sources of BMP ligands, sufficient to re-establish D-V pattern, inducing epidermis at a distance. (Adapted, with permission, from Reversade and De Robertis 2005 [ (C) Elsevier].)

patterning is dependent on the interaction in the extracellular space of BMP ligands, receptors, BMP antagonists, metalloproteinases, and other modulators, which are detailed below.

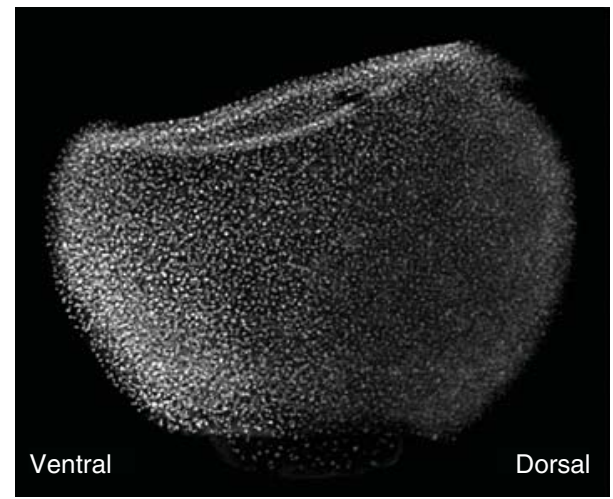

Figure 2. Shape of the BMP morphogenetic gradient in the Xenopus mid-gastrula. Whole-mount staining with phospho-SMAD1/5/8 antibody at midgastrula stage. The ventral center displays high levels of nuclear staining, whereas less staining is detected on the dorsal side.

\section{BMP Ligands}

The main BMP ligands involved in Xenopus $\mathrm{D}-\mathrm{V}$ patterning are BMP2, BMP4, BMP7, and ADMP (antidorsalizing morphogenetic protein). Indeed, only when all four of them are depleted with antisense morpholino oligos does the morphogenetic field collapse, causing ubiquitous neural induction throughout the ectoderm (Fig. 1C) (Reversade and De Robertis 2005). BMP ligands belong to the TGF- $\beta$ superfamily of growth factors (Schmierer and Hill 2007). The mature protein presents a cystine knot domain and is secreted as covalently bound homo- or hetero-dimers. BMP4 and BMP7 are expressed in the ventral center where they induce high BMP signaling. Although counterintuitive, two BMPs, ADMP (Moos et al. 1995) and BMP2 (Inomata et al. 2008), are expressed in the dorsal side where BMP signaling is low. When ventral BMPs are depleted, ADMP and BMP2 expression increases, creating a self-regulative feedback loop in the dorsal side (Reversade and De Robertis 2005; Inomata et al. 
J.-L. Plouhinec and E.M. De Robertis

A

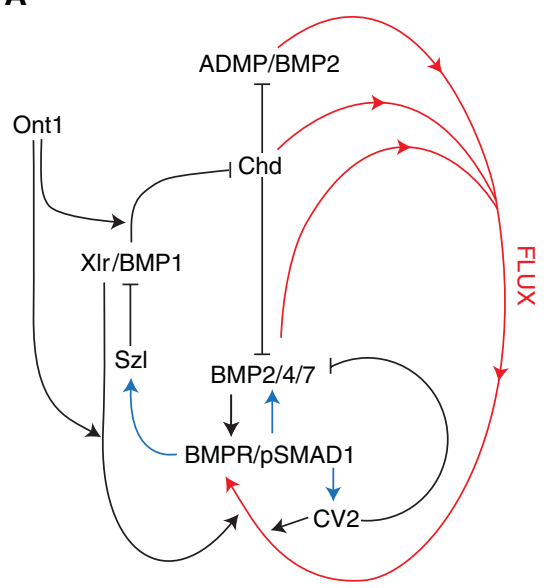

B

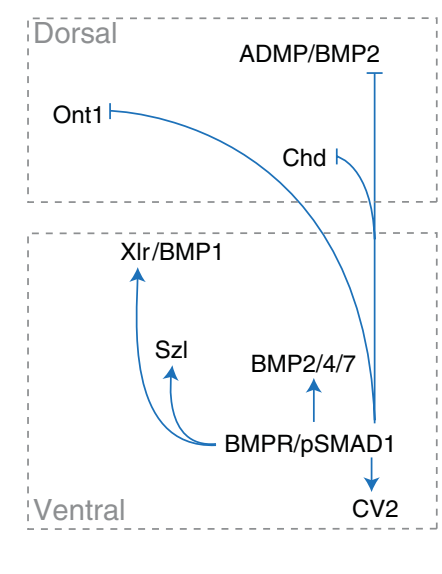

Figure 3. A biochemical pathway of secreted proteins that regulate $\mathrm{D}-\mathrm{V}$ patterning in Xenopus. Black arrows indicate direct protein-protein interactions, blue arrows transcriptional regulation, and red arrows the flux of Chordin/BMP complexes toward the ventral side of the frog gastrula. (A) The dorsal center secretes Chordin (Chd), a BMP antagonist that binds BMP2/4/7 and ADMP, and transports them to the ventral side. The transport and release of BMPs is regulated by tolloid proteases (Xlr/BMP1), in cooperation with the scaffold protein Ont1, and inhibited by Sizzled (Szl). The ventral center secretes CV2, a protein that can inhibit BMPs at high doses, but also promote the flow of Chd/BMP complexes by concentrating them on the ventral side. BAMBI and Twisted gastrulation (Tsg) have been omitted for simplicity. (B) Opposite transcriptional regulation of dorsal and ventral centers by BMP signaling. BMP receptors (BMPR) phosphorylate SMAD1/5/8, repressing dorsal genes (Chd, ADMP, BMP2, and Ont1) and activating ventral genes (BMP2/4/7, CV2, Szl, Xlr). This network enables the D-V morphogenetic field to self-regulate by adjusting levels of BMP signaling on the dorsal and ventral side: At low BMP levels, ADMP and Ont1 are up-regulated, increasing BMP signaling and Chd degradation; at high BMP levels, CV2 and Szl are up-regulated, inhibiting BMPs and stabilizing Chd.

2008), which is capable of promoting BMP signaling at considerable distances from the organizer (Fig. 1F). The BMP homologs in the Drosophila early embryo are called Decapentaplegic (Dpp) and Screw (Scw).

\section{RECEPTORS AND INTRACELLULAR SIGNALING}

The receptors for BMP ligands are transmembrane proteins featuring a cysteine-rich extracellular domain and a conserved intracellular serine/threonine kinase domain (Schmierer and Hill 2007). They belong to two different classes, the type I receptors BMPR-Ia and BMPR-Ib (corresponding to Thickveins in Drosophila) and ALK2 (homologous to Saxophone in Drosophila), and the type II receptors (called Punt in Drosophila). On binding of the ligand, BMP receptors form an heterotetrameric active receptor complex. The Sog-mediated transport of BMPs in Drosophila involves exclusively Dpp/Scw heterodimers (Shimmi et al. 2005). When these heterodimers are released in the dorsal side of the embryo, Scw recruits Saxophone and Dpp recruits Thickveins, resulting in a heterotetrameric receptor complex that generates the peak activity required for amnioserosa formation (Shimmi et al. 2005). A similar situation may exist in zebrafish, in which mutations in BMP2b or BMP7 cause identical and very potent low-BMP (dorsalized) phenotypes (Schmid et al. 2000).

The active receptor complex phosphorylates the SMAD1/5/8 transcription factors at their C-terminus, which triggers their accumulation in the nucleus and subsequent activation of target genes. Thus, nuclear phosphorylated 
Systems Biology of the Morphogenetic Gradient of the Xenopus Gastrula

SMADs (pSMAD1/5/8) provide a direct readout of BMP activity in the embryo. Phosphorylated Smads have been shown to form dynamic D-V gradients in many species including Drosophila (Dorfman and Shilo 2001; Ross et al. 2001; Sutherland et al. 2003), Xenopus (Faure et al. 2000; Schohl and Fagotto 2002; Fig. 2), and zebrafish (Tucker et al. 2008).

\section{Extracellular BMP Antagonists}

\section{Chordin and Noggin}

To regulate the levels of $\mathrm{BMP}$ signaling, the embryo secretes a cocktail of inhibitors, which generate the signaling gradient. The dorsal Spemann organizer expresses two main inhibitors, Noggin and Chordin (Smith and Harland 1992; Sasai et al. 1994). They both bind to BMPs in the extracellular space and prevent their interaction with the BMP receptors (Holley et al. 1996; Piccolo et al. 1996; Zimmerman et al. 1996). Noggin consists of a cystine knot and could therefore be evolutionarily related to BMPs (Groppe et al. 2002). Chordin contains four cysteine rich (CR) domains that can bind BMPs and are present in many other BMP- and TGF- $\beta$-binding molecules (Garcia Abreu et al. 2002). Xenopus or zebrafish embryos depleted for Chordin display an enlarged ventral center, consistent with an inhibitory role of Chordin on the BMP pathway (Schulte-Merker et al. 1997; Oelgeschläger et al. 2003). Chordin is an essential component of the organizer, because Chordin morpholinos completely block the inductive activity of Spemann-organizer grafts (Oelgeschläger et al. 2003). Its Drosophila homolog, Sog (Short Gastrulation), also inhibits BMP activity (Ferguson and Anderson 1992; François et al. 1994), and diffuses from its ventral site of synthesis forming a D-V gradient (Srinivasan et al. 2002). The diffusion of Sog is required for the accumulation of Dpp/ Scw heterodimers on the dorsal side of the Drosophila embryonic blastoderm to achieve peak BMP signaling (Ashe and Levine 1999; Shimmi et al. 2005).

\section{BAMBI}

Another extracellular BMP antagonist is BAMBI (BMP and activin membrane bound inhibitor), a decoy transmembrane BMPRI-like receptor that lacks a functional kinase domain (Onichtchouk et al. 1999). BAMBI can bind to BMPs and to BMPR-II to form a nonproductive complex that cannot trigger BMP signaling. Therefore, BAMBI regulates the availability of both extracellular BMPs and its type II receptors. BAMBI is expressed in the ventral center as a part of the BMP4 synexpression group (Niehrs and Pollet 1999), where it acts as a feedback inhibitor in the ventral center. Although BAMBI has been lost from the Drosophila genome, it plays a key role in the regulation of the $\mathrm{D}-\mathrm{V}$ signaling gradient of the flour beetle Tribolium (Nunes da Fonseca et al. 2008).

\section{Chordin Proteases and Their Regulators}

\section{Tolloids}

Tolloids (Tld) belong to the astacin family of extracellular zinc metalloproteases. The first tolloid was purified together with BMP growth factors from decalcified bone extracellular matrix (Wozney et al. 1988) and designated BMP1. It was subsequently found that a related protease was a key element in the regulation of $\mathrm{D}-\mathrm{V}$ patterning in Drosophila (Shimell et al. 1991). Two of the three vertebrate paralogs, BMP1 and Xolloid-related (Xlr), are expressed during gastrulation in Xenopus, whereas the third, Xolloid, is expressed at higher levels at neurula stages (Dale et al. 2002). Although BMP1 is expressed uniformly, the expression of Xlr is restricted to the ventral center under the positive regulation of BMPs (Dale et al. 2002; Inomata et al. 2008).

Tolloids cleave Chordin/Sog at two specific cleavage sites, with a higher efficiency when Chordin/Sog is bound to BMP ligands and Tsg (Marqués et al. 1997; Piccolo et al. 1997; Larraín et al. 2001; Shimmi and O'Connor 2003). This cleavage produces Chordin fragments with a lower affinity for BMPs. As shown by Piccolo et al. (1997), this allows 
previously inactive BMP4/BMP7 heterodimers to be released from Chordin to signal again. Tolloid proteolysis is the key switch that controls the inhibitory function of Chordin. Recent studies of two additional secreted factors, Sizzled and Ont1, have shown that tolloid proteolytic activity is tightly regulated.

\section{Sizzled}

Sizzled (Szl) is a secreted frizzled related protein (sFRP) expressed in the ventral center (Salic et al. 1997) that, contrary to other sFRPs, lacks anti-Wnt activity (Collavin and Kirschner 2003). Early studies of Ogon, a point mutation in the zebrafish $S z l$ gene, as well as morpholino knockdown in Xenopus, showed that Szl displayed a Chordin-dependent (Yabe et al. 2003) anti-BMP activity (Collavin and Kirschner 2003; Martyn and Schulte-Merker 2003). It has now been established that Szl functions as a competitive inhibitor of tolloid metalloproteinases (Lee et al. 2006; Muraoka et al. 2006). Szl acts as a feedback inhibitor of the ventral center by down-regulating Chordin degradation in the embryo (Fig. 3).

\section{Ont1}

Ont1 is a gene belonging to the olfactomedin family of proteins, which are composed of a coiled-coil domain and an olfactomedin (Olf) domain (Zeng et al. 2005; Sakuragi et al. 2006). At least ten related olfactomedin genes were known to exist in vertebrates, but their function was unknown. In Xenopus, Ont1 is expressed broadly in the dorsal mesoderm and negatively regulated by BMP signaling (Inomata et al. 2008). A recent study has uncovered its role in enhancing the robustness of D-V patterning by regulating the activity of the frog organizer (Inomata et al. 2008). At the molecular level, Ont1 behaves as an adaptor protein that brings together Chordin and Tolloids, forming a complex that promotes the degradation of Chordin. The coiled-coil domain binds to Xlr/BMP1 and the Olf domain binds to the CR2-CR4 region of Chordin. Ont1 knockdown increases the sensitivity of the
Xenopus embryo to microinjection of Chordin. Therefore, Ont1 is a novel regulator of Chordin levels in the embryo (Fig. 3), which leads to Chordin degradation by increasing Tolloid activity.

\section{Additional Extracellular Modulators}

\section{Twisted Gastrulation}

Twisted gastrulation (Tsg) is an evolutionarily conserved context-dependent modulator of BMP signaling that is present in both Drosophila (Mason et al. 1994) and vertebrates (Oelgeschläger et al. 2000). It is secreted by the ventral center and can bind both to Chordin and BMPs to form a stable ternary complex unable to bind BMP receptors (Oelgeschläger et al. 2000; Chang et al. 2001; Ross et al. 2001; Scott et al. 2001). However, the Chd/BMP/Tsg complex is more sensitive to cleavage by Tolloid proteases, and Tsg can efficiently compete with Chordin fragments for the binding of BMPs (Larraín et al. 2001; Scott et al. 2001). Therefore, after Chordin/BMP/Tsg complexes are cleaved by Tolloid at the Chordin sites, the activity of Tsg switches from a BMP inhibitory cofactor to a pro-BMP factor. In zebrafish, the overall function of Tsg is to promote BMP signaling, because its depletion with antisense morpholinos causes dorsalization of the embryo (Little and Mullins 2004; Xie and Fisher 2005), contrary to initial reports (Ross et al. 2001). In Xenopus embryos depleted of Chordin and Crossveinless-2, there is an absolute requirement for Tsg for BMP signaling to occur (Ambrosio et al. 2008). Probably one function of Tsg is to keep heterodimers between BMP2/4 and ADMP or BMP7 in a soluble state in the extracellular space. Indeed, in Drosophila, Sog requires Tsg to detach $\mathrm{Dpp} / \mathrm{Scw}$ heterodimers from the extracellular matrix (Wang et al. 2008).

\section{Crossveinless-2}

Crossveinless-2 (CV2) is a secreted protein required for the formation of the Drosophila wing crossveins (Conley et al. 2000). It is conserved in vertebrates (Coffinier et al. 2002) 
and possesses five Chordin-like CR domains, as well as a von Willebrand factor D (vWF-D) domain. CV2 is autocatalytically processed in the secretory pathway into two fragments containing respectively the CRs and the vWF-D domains, linked by a disulfide bridge (Binnerts et al. 2004; Kamimura et al. 2004; Ambrosio et al. 2008). The CV2 gene is expressed in the ventral center in Xenopus under the positive control of BMPs, and has been shown in a diverse range of settings to be able to both promote and antagonize BMP signaling (Ikeya et al. 2006; Rentzsch et al. 2006; Ambrosio et al. 2008; Zakin et al. 2008). In Drosophila, cv2 is expressed in sites of high BMP signaling, such as the crossveins and the dorsal third of the blastoderm (Serpe et al. 2008). Recent structural, biochemical, and mathematical studies (Ambrosio et al. 2008; Serpe et al. 2008; Zhang et al. 2008) suggest that CV2 is a key component of the Chd/Sog biochemical pathway, in which it helps ensure that a flux of Chd/Tsg/BMP towards the regions of peak BMP signaling is maintained.

\section{REACHING PEAK LEVELS OF BMP SIGNALING}

As shown in Figure 4, a biochemical pathway of the molecular interactions occurring in the ventral center of Xenopus (or dorsal-most stripe of Drosophila) is starting to emerge. Several properties are worth noting.

First, CV2 contains a heparan sulfate proteoglycan (HSPG) binding site in its vWF-D domain (Rentzsch et al. 2006). In Drosophila wing discs, the binding of CV2 to the HSPG glypican Dally has been shown to prevent it from diffusing beyond two or three cell diameters from its site of synthesis (Serpe et al. 2008). In the mouse, CV2 protein also remains anchored at its site of synthesis in prevertebral cartilages (Zakin et al. 2008). Thus, CV2 protein remains concentrated on the surface of cells in which BMP signaling is high.

Second, the fact that CV2 is tethered to the cell membrane via the glycosyl phosphatidyl inositol (GPI) anchor of Dally (Serpe et al.
2008) presents interesting opportunities for the endocytosis of CV2. GPI anchors are lipidic and bind preferentially to cholesterolrich regions of membranes, which in turn are associated with endocytosis via the caveoli pathway, which is generally followed by intracellular degradation (Fig. 4).

Third, structural studies have shown that the first CR domain of CV2 interacts with BMP dimers in a 2:1 ratio, forming inhibitory BMP-CV2 complexes (Zhang et al. 2008). CV2 also binds Tsg, and becomes a better BMP antagonist in its presence (Ambrosio et al. 2008).

Fourth, CV2 binds to the Thickveins type I receptor via its CR domains (Serpe et al. 2008) (Fig. 4). This has led to the proposal of a model of facilitated transfer, in which the vicinity with $\mathrm{CV} 2$ promotes the transfer of BMP from CV2 to Thickveins. Using mathematical modeling, it has been shown that BMP signaling can be promoted at low CV2 concentrations in a ligand-specific manner and is inhibited at high concentrations of CV2 that antagonize the formation of active receptor-ligand complexes (Serpe et al. 2008).

Finally, Ambrosio et al. (2008) discovered that CV2 is a Chordin-binding protein. Full-length Chordin binds to CV2 with an affinity of $1 \mathrm{nM}$ in biochemical assays. Interestingly, CV2 displays an even higher affinity for Chordin complexed with BMP4 and for Chordin protein fragments after cleavage by Tolloids (Ambrosio et al. 2008).

CV2 may be involved in the removal of spent Chordin fragments from the extracellular space (Fig. 4), because CV2 depletion causes the Xenopus embryo to become hypersensitive to microinjected Chordin protein (Ambrosio et al. 2008). Chordin is secreted in prodigious amounts in the Xenopus gastrula, at an estimated concentration of $30 \mathrm{nM}$ in the extracellular space if it were uniformly distributed (Lee et al. 2006). Therefore, removing extracellular Chordin should be essential to maintaining the $\mathrm{D}-\mathrm{V}$ flux of morphogens. The flow of epitope-tagged BMP4 from dorsal to ventral has been recently directly shown in Xenopus, and shown to require Chordin (Ben-Zvi et al. 2008). 
J.-L. Plouhinec and E.M. De Robertis

A
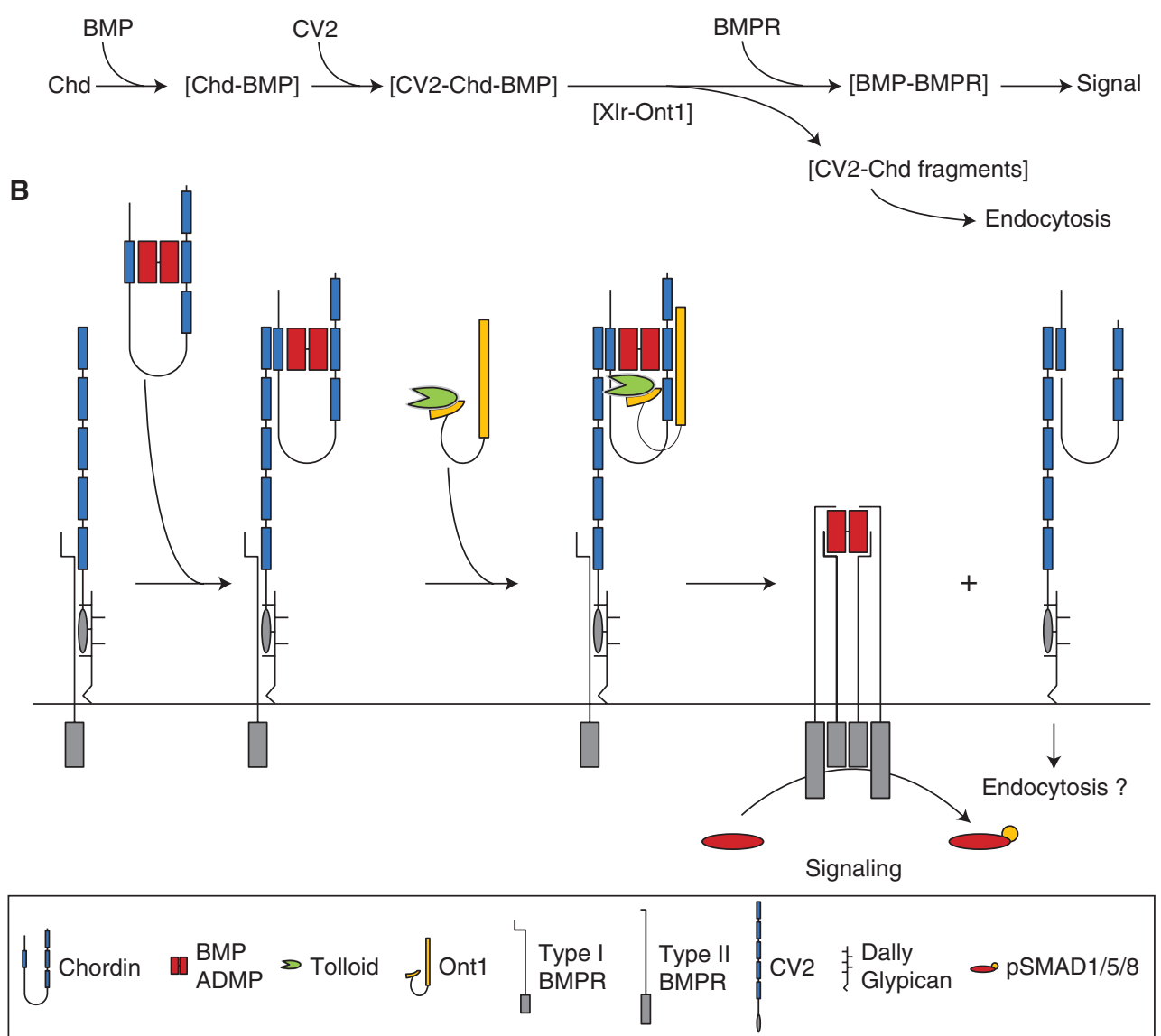

Figure 4. Model of the regulation of the flow of Chordin/BMP complexes by CV2. (A) Biochemical pathway depicting the regulation of the BMP flow by CV2. (B) Model of the molecules participating in the signaling pathway in the ventral center. The interaction of CV2 with membrane-anchored glypican/Dally restricts CV2 diffusion and keeps it localized to the ventral center. CV2 binds with high affinity to Chd/BMP complexes diffusing from more dorsal regions of the embryo. The Chd/BMP complex (which also includes Tsg, not shown) is then cleaved by tolloid proteases (with the help of the scaffold protein Ont1) while still bound to the CV2 complex, which also contains BMP Type I receptors. This facilitates the binding of BMPs released by tolloid to their Type I receptors, whereas Chd fragments remain bound to CV2 and may be cleared by endocytosis. In this model, the pro-BMP activity of CV2 is caused by the flow of Chd/BMP complexes to the ventral side, their release by Tolloid, and the clearing of Chordin residual fragments (which are still able to bind BMPs via their CR modules).

In the absence of tolloids, CV2 would capture Chordin-BMP complexes and inhibit signaling (anti-BMP effect). In the diagram shown in Figure 4, we propose that ChordinTsg-BMP complexes bound to CV2 might be key substrates for the cleavage by Tolloid (in collaboration with the scaffold protein olfactomedin/Ont 1). In these circumstances, the presence of Thickveins/BMPR-Ia in the same protein complex would greatly facilitate transfer of the liberated BMPs to their cognate receptors followed by signaling, as proposed by Serpe et al. (2008). In this view, the pro-BMP activity of CV2 would be caused by concentrating Chordin/Tsg/BMP complexes diffusing from other regions of the embryo in which BMP signaling levels are lower (Ambrosio et al. 2008). This property of CV2 might also explain the 
positive feedback mechanism in the Drosophila amnioserosa by which Dpp/Scw signaling, which is initially more diffuse, becomes focused to a narrow band of cells in the dorsal midline (Wang and Ferguson 2005; Umulis et al. 2006). This bistability in signaling levels is a function of previous exposure to BMP signaling. Although it is not known at present whether Drosophila $c v 2$ is transcriptionally controlled by BMP signaling, its mRNA is expressed in the dorsal third of the blastoderm (Serpe et al. 2008), and is a prime candidate to contribute to the generation of the bistability switch (Umulis et al. 2006).

\section{MATHEMATICAL MODELING OF THE EXTRACELLULAR NETWORK}

D-V patterning requires an extracellular network of proteins regulated by opposite transcriptional controls (Fig. 3) to create a robust pSMAD gradient that determines cell type differentiation. These proteins, their interactions, and regulation have been uncovered by genetic, cloning, and biochemical studies, which have provided many insights into the key processes that underlie the behavior of the system. However, as knowledge of the pathway improves and its complexity is uncovered, quantitative mathematical models are needed to analyze the properties of the network (Reeves et al. 2006; Lander 2007; Ibañes and Belmonte 2008). The eventual goal is to understand the properties of the entire system that mediates the self-regulation of a morphogenetic field. These insights are difficult to achieve by intuition alone and require mathematical analysis (Plouhinec and De Robertis 2007). Partial differential equations (PDE) provide a natural way to model the spatio-temporal evolution of diffusing molecules (Turing 1952; Gierer and Meinhardt 1972).

British mathematician Alan Turing proposed in 1952 that pattern formation in development might be explicable by a system of chemical substances that react together and diffuse between cells following the ordinary laws of thermodynamics (the law of mass action and Fick's law of diffusion). He coined the term "morphogen" for these mysterious chemicals and formulated reaction-diffusion equations of the type shown at the top of Figure 5. The equation describes the changes in concentration of a morphogen $\mathrm{C}$ over time $(t)$ as a function of its diffusion, degradation, and synthesis. We are constructing an "electronic frog" model that includes most of the interacting components shown in Figure 3A, as well as their transcriptional regulation. The evolution of each molecular component (protein or complex) is defined by a single equation. The PDE at the bottom of Figure 5 describes how the concentration of Chordin changes over time in the embryo. The multiple equations in the model are intertwined because they share common chemical reactions. This mathematical model is able to self-regulate the pattern of a BMP gradient (J.-L. Plouhinec and E.M. De Robertis, unpubl.). Recently, a simplified reaction-diffusion model has shown that the flow of BMP is an essential requirement for the generation of a stable and scalable BMP gradient (Ben-Zvi et al. 2008).

The combination of mathematical modeling and experimental approaches has already proven very fruitful in the study of Drosophila D-V patterning (Eldar et al. 2002; Mizutani et al. 2005; Shimmi et al. 2005; Kicheva and González-Gaitán 2008; Serpe et al. 2008) and is now being extended to vertebrates (Zhang et al. 2007b; Ben-Zvi et al. 2008). These studies have uncovered several features of the $\mathrm{D}-\mathrm{V}$ patterning network that account for its robustness.

At the extracellular level, Chordin/Sog not only inhibits BMPs by binding to them and preventing interaction with their receptors, but in combination with Tolloid proteases drives a flow of BMPs to the ventral side (Piccolo et al. 1997; Ashe and Levine 1999; Eldar et al. 2002; Srinivasan et al. 2002). Mathematical modeling has suggested that the flux of BMPs mediated by Chordin/Sog is crucial for the robustness of the BMP gradient (Eldar et al. 2002; Ben-Zvi et al. 2008). It is interesting to note that Chordin is important but not crucial to pattern the mouse gastrula (Bachiller et al. 2000, 2003), indicating that the slower developing 
J.-L. Plouhinec and E.M. De Robertis

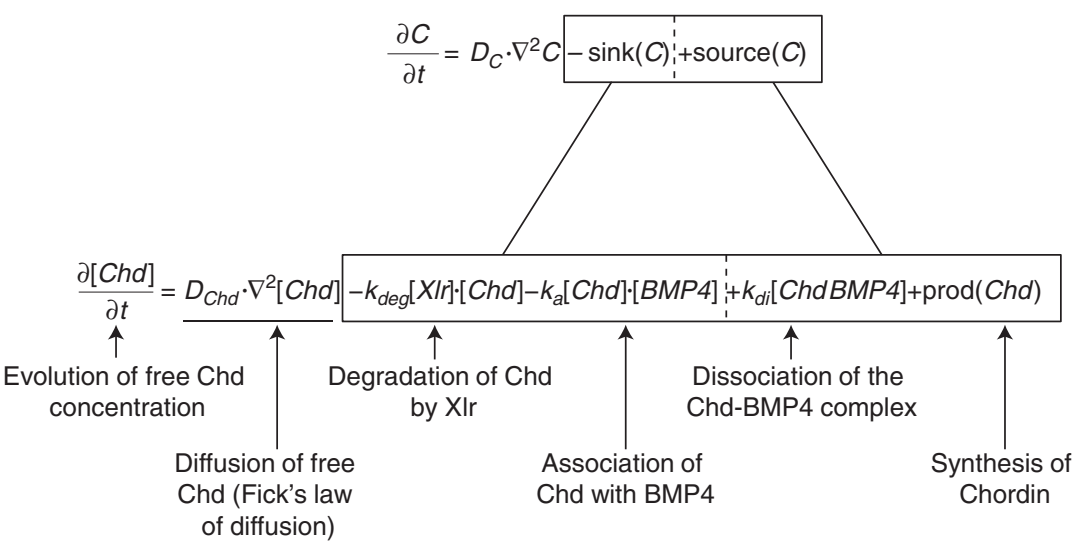

Figure 5. Partial differential equation describing the evolution of free Chordin concentration in a minimal model of dorsal-ventral patterning. The equation shown on top is a simplified version, based on the reaction-diffusion equations proposed by Turing in 1952. The biochemical model shown below includes Chordin (Chd) and BMP4, which can form a Chd-BMP4 complex (Chd+BMP4 $\leftrightarrow$ Chd-BMP4), as well as the Xolloid-related protease (Xlr), which can degrade Chd and Chd-BMP4 (Chd $+\mathrm{Xlr} \rightarrow \mathrm{Xlr}$; Chd-BMP4+Xlr $\rightarrow \mathrm{BMP} 4+\mathrm{Xlr})$. Chd is produced dorsally, and the protein and its complexes can diffuse. This model can be described mathematically by a system of intertwined reaction-diffusion partial differential equations (PDE), each equation describing the evolution of the concentration of one component of the system (protein or complex) over time. The evolution of the concentration (shown here for free Chordin) takes into account the diffusion, as well as the reactions that lead to the synthesis and degradation of the component. These equations are generally applicable to many patterning systems. They enable the use of mathematical and numerical tools to analyze the behavior of a self-regulating morphogenetic gradient. $\nabla^{2}$ is the second derivative in space, $\mathrm{D}_{\mathrm{Chd}}$ is the diffusion rate of free $\mathrm{Chd}$, and $\mathrm{k}_{\mathrm{deg}}, \mathrm{k}_{\mathrm{a}}$, and $\mathrm{k}_{\mathrm{di}}$ the degradation, association, and dissociation rates of the reactions involving free Chd.

mammalian embryos may have evolved new strategies. Perhaps the need for self-regulation is decreased when the embryo develops at a constant temperature, or when epiboly movements over a reserve of yolk are no longer required.

The robustness is encoded in the regulatory topology of the D-V field that generates the BMP activity gradient. The field is patterned by two synexpression groups, the dorsal Chordin organizer, and the ventral BMP center, each one expressing a coherent set of BMP ligands and antagonists (De Robertis and Kuroda 2004). The two centers are under opposite transcriptional regulation by BMP signaling (Fig. 3B), which ensure their mutual exclusivity and location at opposite poles of the embryo (Reversade and De Robertis 2005). Each center is self-regulative, because it secretes antagonists of its own activity: BMPs and anti-Chordin proteins (Ont1) on the dorsal side, and anti-BMPs (CV2, BAMBI) and proChordin (Sizzled) molecules on the ventral side (Fig. 3A). This feedback adjusts the level of BMP activity on the dorsal and ventral side, a property essential for the scaling of the gradient with size; for example, when an embryo is cut in half or subjected to a Spemann graft that results in two Siamese twins of the same size (Reversade and De Robertis 2005; Lee at al. 2006). Finally, each center regulates at a distance the activity of the opposite center, as evidenced by the fact that transplantation of either the dorsal center or the ventral center can regenerate the D-V gradient (Fig. 1) (Reversade and De Robertis 2005). This last property makes the D-V field robust to perturbations of BMP levels, which likely is key to its regeneration abilities.

\section{PROSPECTS}

The time is now ripe for a fruitful interaction between experimental embryology and theoretical modeling to reverse engineer the $\mathrm{D}-\mathrm{V}$ 
morphogenetic field. Physiochemical properties of the interactions between components, such as dissociation constants $\left(\mathrm{K}_{\mathrm{D}}\right)$ and enzymatic Michaelis constants $\left(\mathrm{K}_{\mathrm{m}}\right)$, have been determined (see, e.g., Piccolo et al. 1996; Dyson and Gurdon 1998; Lee et al. 2006; Zhang et al. 2007a; Ambrosio et al. 2008). It is feasible to image and quantify the gradient of BMP activity (Fig. 2) (Tucker et al. 2008), and the Xenopus embryo provides ways to manipulate the gradient experimentally (see, e.g., McDowell et al. 1997; Oelgeschläger et al. 2003; Reversade and De Robertis 2005).

Many important unanswered questions concerning the Xenopus gastrula patterning gradient remain. Given the short time frame for the establishment of a $\mathrm{D}-\mathrm{V}$ gradient in a $1.2 \mathrm{~mm}$ Xenopus embryonic field of about 10,000 cells (less than $4 \mathrm{~h}$ at $22^{\circ} \mathrm{C}$ from blastula to mid-gastrula), has the BMP gradient reached a steady state or is the gradient interpreted whereas still in the transient phase? This could affect which molecular process is important in the formation of the gradient (Lander 2007). There is, for example, evidence that in the case of the activin morphogen the gradient is interpreted dynamically (Gurdon et al. 1995). Given that a network consisting of only BMP, Sog, and Tolloid can be modeled to generate a gradient of BMP activity (Eldar et al. 2002), how useful are the additional extracellular feedback regulators for the control of the endogenous gradient? Indeed, some of them have been lost during evolution (BAMBI was lost in Drosophila but is found in vertebrates and Tribolium) or are restricted to a subgroup of bilateria (Sizzled has so far only been found in vertebrates; J.-L. Plouhinec and E.M. De Robertis, unpubl.). How is $\mathrm{D}-\mathrm{V}$ positional information integrated with other signaling pathways to provide a full coordinate system for cells in the embryo? SMAD transcription factors provide an interesting platform because they can integrate BMP, Wnt, and FGF signals through multiple phosphorylation sites that regulate their activation and the duration of the signal (Fuentealba et al. 2007; Eivers et al. 2008). Finally, how is D-V patterning integrated during the extensive morphogenetic movements in the Xenopus gastrula? Two observations of research provide interesting starting points to investigate this last question: BMPs regulate the migration of cells in the zebrafish gastrula (von der Hardt et al. 2007; Tucker et al. 2008) and also control cell proliferation in the Drosophila wing (Rogulja and Irvine 2005).

A century of research in amphibian embryos has uncovered the outlines of the extracellular machinery that generate and self-regulate the $\mathrm{D}-\mathrm{V}$ axis (timeline in De Robertis 2006). It started with Spemann with the discovery of embryonic induction and fields, concepts that borrowed heavily on physics, the dominant science at the time. In the 1990s, a phase of molecular discovery of the identified components of the machinery showed their interactions in chemical terms. Embryology is now returning to a systems approach, based on mathematics and physics, to understand the behavior of the whole.

\section{ACKNOWLEDGMENTS}

We thank the members of the De Robertis lab for their comments on the manuscript. Our work is supported by National Institute of Health grant HD21502-22 and the Howard Hughes Medical Institute.

\section{REFERENCES}

Ambrosio AL, Taelman VF, Lee HX, Metzinger CA, Coffinier C, De Robertis EM. 2008. Crossveinless-2 is a BMP feedback inhibitor that binds Chordin/BMP to regulate Xenopus embryonic patterning. Dev Cell 15: $248-260$.

Ashe HL, Levine M. 1999. Local inhibition and long-range enhancement of Dpp signal transduction by Sog. Nature 398: 427-431.

Bachiller D, Klingensmith J, Kemp C, Belo JA, Anderson RM, May SR, McMahon JA, McMahon AP, Harland R, Rossant J, et al. 2000. The organizer factors Chordin and Noggin are required for mouse forebrain development. Nature 403: 658-661.

Bachiller D, Klingensmith J, Schneyder N, Tran U, Anderson R, Rossant J, De Robertis EM. 2003. The role of chordin/Bmp signals in mammalian pharyngeal development and DiGeorge syndrome. Development 130: $3567-3578$. 


\section{J.-L. Plouhinec and E.M. De Robertis}

Ben-Zvi D, Shilo B, Fainsod A, Barkai N. 2008. Scaling of the BMP activation gradient in Xenopus embryos. Nature 453: $1205-1211$.

Binnerts ME, Wen X, Canté-Barrett K, Bright J, Chen HT, Asundi V, Sattari P, Tang T, Boyle B, Funk W, et al. 2004. Human Crossveinless-2 is a novel inhibitor of bone morphogenetic proteins. Biochem Biophys Res Commun 315: 272-280.

Chang C, Holtzman DA, Chau S, Chickering T, Woolf EA, Holmgren LM, Bodorova J, Gearing DP, Holmes WE, Brivanlou AH. 2001. Twisted gastrulation can function as a BMP antagonist. Nature 410: 483-487.

Coffinier C, Ketpura N, Tran U, Geissert D, De Robertis EM. 2002. Mouse Crossveinless-2 is the vertebrate homolog of a Drosophila extracellular regulator of BMP signaling. Mech Dev 119: S179-S184.

Collavin L, Kirschner MW. 2003. The secreted Frizzledrelated protein Sizzled functions as a negative feedback regulator of extreme ventral mesoderm. Development 130: $805-816$.

Conley C, Silburn R, Singer M, Ralston A, Rohwer-Nutter D, Olson D, Gelbart W, Blair S. 2000. Crossveinless 2 contains cysteine-rich domains and is required for high levels of BMP-like activity during the formation of the cross veins in Drosophila. Development 127: 3947-3959.

Crick F. 1970. Diffusion in embryogenesis. Nature 225: 420-422.

Dale L, Evans W, Goodman SA. 2002. Xolloid-related: A novel BMP1/Tolloid-related metalloprotease is expressed during early Xenopus development. Mech Dev 119: 177-190.

De Robertis EM. 2006. Spemann's organizer and selfregulation in amphibian embryos. Nat Rev Mol Cell Biol 7: 296-302.

De Robertis EM. 2008. Evo-Devo: Variations on ancestral themes. Cell 132: 185-195.

De Robertis EM, Kuroda H. 2004. Dorsal-ventral patterning and neural induction in Xenopus embryos. Annu Rev Cell Dev. Biol. 20: 285-308.

De Robertis EM, Sasai Y. 1996. A common plan for dorsoventral patterning in Bilateria. Nature 380: 37-40.

De Robertis EM, Morita EA, Cho KWY. 1991. Gradient fields and homeobox genes. Development 112: 669-678.

Dorfman R, Shilo B. 2001. Biphasic activation of the BMP pathway patterns the Drosophila embryonic dorsal region. Development 128: 965-972.

Driever W, Nüsslein-Volhard C. 1988. The bicoid protein determines position in the Drosophila embryo in a concentration-dependent manner. Cell 54: 95-104.

Dyson S, Gurdon JB. 1998. The interpretation of position in a morphogen gradient as revealed by occupancy of activin receptors. Cell 93: 557-568.

Eivers E, Fuentealba LC, De Robertis E. 2008. Integrating positional information at the level of Smad1/5/8. Curr Opin Genet Dev 18: 304-310.

Eldar A, Dorfman R, Weiss D, Ashe H, Shilo B, Barkai N. 2002. Robustness of the BMP morphogen gradient in Drosophila embryonic patterning. Nature 419: 304-308.

Faure S, Lee M, Keller T, ten Dijke P, Whitman M. 2000. Endogenous patterns of TGF- $\beta$ superfamily signaling during early Xenopus development. Development 127: 2917-2931.

Ferguson E, Anderson K. 1992. Localized enhancement and repression of the activity of the TGF- $\beta$ family member, decapentaplegic, is necessary for dorsal-ventral pattern formation in the Drosophila embryo. Development 114: 583-597.

François V, Solloway M, O’Neill JW, Emery J, Bier E. 1994. Dorsal-ventral patterning of the Drosophila embryo depends on a putative negative growth factor encoded by the short gastrulation gene. Genes Dev 8: 2602-2616.

Fuentealba LC, Eivers E, Ikeda A, Hurtado C, Kuroda H, Pera EM, De Robertis EM. 2007. Integrating patterning signals: Wnt/GSK3 regulates the duration of the BMP/ Smad1 signal. Cell 131: 980-993.

Garcia Abreu J, Coffinier C, Larraín J, Oelgeschläger M, De Robertis EM. 2002. Chordin-like CR domains and the regulation of evolutionarily conserved extracellular signaling systems. Gene 287: 39-47.

Gierer A, Meinhardt H. 1972. A theory of biological pattern formation. Kybernetik 12: 30-39.

Gregor T, Wieschaus EF, McGregor AP, Bialek W, Tank DW. 2007. Stability and nuclear dynamics of the Bicoid morphogen gradient. Cell 130: 141-152.

Groppe J, Greenwald J, Wiater E, Rodriguez-Leon J, Economides AN, Kwiatkowski W, Affolter M, Vale WW, Belmonte JC, Choe S. 2002. Structural basis of BMP signalling inhibition by the cystine knot protein Noggin. Nature 420: 636-642.

Gurdon JB, Mitchell A, Mahony D. 1995. Direct and continuous assessment by cells of their position in a morphogen gradient. Nature 376: 520-521.

Heemskerk J, DiNardo S. 1994. Drosophila hedgehog acts as a morphogen in cellular patterning. Cell 76: 449-460.

Holley SA, Jackson PD, Sasai Y, Lu B, De Robertis EM, Hoffman FM, Ferguson EL. 1995. A conserved system for dorsal-ventral patterning in insects and vertebrates involving sog and chordin. Nature 376: 249-253.

Holley SA, Neul JL, Attisano L, Wrana JL, Sasai Y, O'Connor MB, De Robertis EM, Ferguson EL. 1996. The Xenopus dorsalizing factor noggin ventralizes Drosophila embryos by preventing DPP from activating its receptor. Cell 86: 607-617.

Holm Å. 1952. Experimentelle Untersuchungen über die Entwicklung und Entwicklungsphysiologie des Spinnenembryos. Zool BiDr Uppsala 29: 293-424.

Ibañes M, Belmonte JCI. 2008. Theoretical and experimental approaches to understand morphogen gradients. Mol Syst Biol 4: 176.

Ikeya M, Kawada M, Kiyonari H, Sasai N, Nakao K, Furuta Y, Sasai Y. 2006. Essential pro-BMP roles of crossveinless 2 in mouse organogenesis. Development 133: 4463-4473.

Inomata H, Haraguchi T, Sasai Y. 2008. Robust stability of the embryonic axial pattern requires a secreted scaffold for Chordin degradation. Cell 134: 854-865.

Kamimura M, Matsumoto K, Koshiba-Takeuchi K, Ogura T. 2004. Vertebrate crossveinless 2 is secreted and acts as an extracellular modulator of the BMP signaling cascade. Dev Dyn 230: 434-445. 
Kicheva A, González-Gaitán M. 2008. The Decapentaplegic morphogen gradient: A precise definition. Curr Opin Cell Biol 20: 137-143.

Kondo M. 2007. Bone morphogenetic proteins in the early development of zebrafish. FEBS J. 274: 2960-2967.

Lander AD. 2007. Morpheus unbound: Reimagining the morphogen gradient. Cell 128: 245-256.

Larraín J, Oelgeschläger $M$, Ketpura NI, Reversade B, Zakin L, De Robertis EM. 2001. Proteolytic cleavage of Chordin as a switch for the dual activities of Twisted gastrulation in BMP signaling. Development 128: 4439-4447.

Lee HX, Ambrosio AL, Reversade B, De Robertis E. 2006. Embryonic dorsal-ventral signaling: Secreted Frizzledrelated proteins as inhibitors of Tolloid proteinases. Cell 124: 147-159.

Little SC, Mullins MC. 2004. Twisted gastrulation promotes BMP signaling in zebrafish dorsal-ventral axial patterning. Development 131: 5825-5835.

Marqués G, Musacchio M, Shimell MJ, WunnenbergStapleton K, Cho KWY, O'Connor MB. 1997. Production of a DPP activity gradient in the early Drosophila embryo through the opposing actions of the SOG and TLD proteins. Cell 91: 417-426.

Martyn U, Schulte-Merker S. 2003. The ventralized ogon mutant phenotype is caused by a mutation in the zebrafish homologue of Sizzled, a secreted Frizzled-related protein. Dev Biol 260: 58-67.

Mason ED, Konrad KD, Webb CD, Marsh JL. 1994. Dorsal midline fate in Drosophila embryos requires twisted gastrulation, a gene encoding a secreted protein related to human connective tissue growth factor. Genes Dev 8: 1489-1501.

McDowell N, Zorn A, Crease D, Gurdon J. 1997. Activin has direct long-range signalling activity and can form a concentration gradient by diffusion. Curr Biol 7: 671-681.

Mizutani CM, Nie Q, Wan FY, Zhang Y, Vilmos P, Sousa-Neves R, Bier E, Marsh JL, Lander AD. 2005. Formation of the BMP activity gradient in the Drosophila embryo. Dev Cell 8: 915-924.

Moos M, Wang S, Krinks M. 1995. Anti-dorsalizing morphogenetic protein is a novel TGF- $\beta$ homolog expressed in the Spemann organizer. Development 121: 4293-4301.

Muraoka O, Shimizu T, Yabe T, Nojima H, Bae Y, Hashimoto H, Hibi M. 2006. Sizzled controls dorsoventral polarity by repressing cleavage of the Chordin protein. Nat Cell Biol 8: 329-340.

Niehrs C, Pollet N. 1999. Synexpression groups in eukaryotes. Nature 402: 483-487.

Nunes da Fonseca R, von Levetzow C, Kalscheuer P, Basal A, van deer Zee M, Roth S. 2008. Self-regulatory circuits in dorsoventral axis formation of the short-germ beetle Tribolium castaneum. Dev Cell 14: 605-615.

Oda H, Akiyama-Oda Y. 2008. Differing strategies for forming the arthropod body plan: Lessons from Dpp, Sog and Delta in the fly Drosophila and spider Achaearanea. Dev Growth Differ 50: 203-214.

Oelgeschläger M, Larraín J, Geissert D, De Robertis EM. 2000. The evolutionarily conserved BMP-binding protein Twisted gastrulation promotes BMP signalling. Nature 405: 757-763.
Oelgeschläger M, Kuroda H, Reversade B, De Robertis EM. 2003. Chordin is required for the Spemann organizer transplantation phenomenon in Xenopus embryos. Dev Cell 4: 219-230.

Onichtchouk D, Chen Y, Dosch R, Gawantka V, Delius H, Massagué J, Niehrs C. 1999. Silencing of TGF- $\beta$ signalling by the pseudoreceptor BAMBI. Nature 401: $480-485$.

Piccolo S, Sasai Y, Lu B, De Robertis EM. 1996. Dorsoventral patterning in Xenopus: Inhibition of ventral signals by direct binding of Chordin to BMP-4. Cell 86: 589-598.

Piccolo S, Agius E, Lu B, Goodman S, Dale L, De Robertis EM. 1997. Cleavage of Chordin by Xolloid metalloprotease suggests a role for proteolytic processing in the regulation of Spemann organizer activity. Cell 91: 407-416.

Plouhinec J-L, De Robertis EM. 2007. Systems biology of embryonic morphogens. Mol Biosyst 3: 454-457.

Reeves GT, Muratov CB, Schupbach T, Shvartsman SY. 2006. Quantitative models of developmental pattern formation. Dev Cell 11: 289-300.

Rentzsch F, Zhang J, Kramer C, Sebald W, Hammerschmidt M. 2006. Crossveinless 2 is an essential positive feedback regulator of Bmp signaling during zebrafish gastrulation. Development 133: 801-811.

Reversade B, De Robertis E. 2005. Regulation of ADMP and BMP2/4/7 at opposite embryonic poles generates a self-regulating morphogenetic field. Cell 123: 11471160.

Rogulja D, Irvine KD. 2005. Regulation of cell proliferation by a morphogen gradient. Cell 123: 449-461.

Ross JJ, Shimmi O, Vilmos P, Petryk A, Kim H, Gaudenz K, Hermanson S, Ekker SC, O’Connor MB, Marsh JL. 2001. Twisted gastrulation is a conserved extracellular BMP antagonist. Nature 410: 479-483.

Rusch J, Levine M. 1996. Threshold responses to the dorsal regulatory gradient and the subdivision of primary tissue territories in the Drosophila embryo. Curr Opin Genet Dev 6: 416-423.

Sakuragi M, Sasai N, Ikeya M, Kawada M, Onai T, Katahira T, Nakamura H, Sasai Y. 2006. Functional analysis of chick ONT1 reveals distinguishable activities among olfactomedin-related signaling factors. Mech Dev 123: $114-123$.

Salic A, Kroll K, Evans L, Kirschner M. 1997. Sizzled: A secreted Xwnt8 antagonist expressed in the ventral marginal zone of Xenopus embryos. Development 124: $4739-4748$.

Sasai Y, Lu B, Steinbeisser H, Geissert D, Gont LK, De Robertis EM. 1994. Xenopus chordin: A novel dorsalizing factor activated by organizer-specific homeobox genes. Cell 79: 779-790.

Schier AF, Talbot WS. 2005. Molecular genetics of axis formation in zebrafish. Annu Rev Genet 39: 561-613.

Schmid B, Fürthauer M, Connors SA, Trout J, Thisse B, Thisse C, Mullins MC. 2000. Equivalent genetic roles for $b m p 7 /$ snailhouse and $b m p 2 b /$ swirl in dorsoventral pattern formation. Development 127: 957-967.

Schmierer B, Hill CS. 2007. TGF- $\beta$-SMAD signal transduction: Molecular specificity and functional flexibility. Nat Rev Mol Cell Biol 8: 970-982. 


\section{J.-L. Plouhinec and E.M. De Robertis}

Schohl A, Fagotto F. 2002. $\beta$-catenin, MAPK and Smad signaling during early Xenopus development. Development 129: $37-52$.

Schulte-Merker S, Lee KJ, McMahon AP, Hammerschmidt M. 1997. The zebrafish organizer requires chordino. Nature 387: 862-863.

Scott IC, Blitz IL, Pappano WN, Maas SA, Cho KWY Greenspan DS. 2001. Homologues of Twisted gastrulation are extracellular cofactors in antagonism of BMP signalling. Nature 410: 475-478.

Serpe M, Umulis D, Ralston A, Chen J, Olson DJ, Avanesov A, Othmer H, O'Connor MB, Blair SS. 2008 The BMP-binding protein Crossveinless 2 is a shortrange, concentration-dependent, biphasic modulator of BMP signaling in Drosophila. Dev Cell 14: 940-953.

Shimell MJ, Ferguson EL, Childs SR, O'Connor MB. 1991. The Drosophila dorsal-ventral patterning gene tolloid is related to human bone morphogenetic protein 1. Cell 67: $469-481$.

Shimmi O, O'Connor MB. 2003. Physical properties of Tld, Sog, Tsg and Dpp protein interactions are predicted to help create a sharp boundary in Bmp signals during dorsoventral patterning of the Drosophila embryo. Development 130: 4673-4682.

Shimmi O, Umulis D, Othmer H, O'Connor MB. 2005. Facilitated transport of a $\mathrm{Dpp} / \mathrm{Scw}$ heterodimer by Sog/Tsg leads to robust patterning of the Drosophila blastoderm embryo. Cell 120: 873-886.

Smith WC, Harland RM. 1992. Expression cloning of noggin, a new dorsalizing factor localized to the Spemann organizer in Xenopus embryos. Cell 70: 829-840.

Spemann H, Mangold H. 1924. Über Induktion von Embryonalanlagen durch Implantation artfremder Organisatoren. Dev Genes Evol 100: 599-638.

Srinivasan S, Rashka KE, Bier E. 2002. Creation of a Sog morphogen gradient in the Drosophila embryo. Dev Cell 2: 91-101.

Sutherland DJ, Li M, Liu X, Stefancsik R, Raftery LA. 2003. Stepwise formation of a SMAD activity gradient during dorsal-ventral patterning of the Drosophila embryo. Development 130: 5705-5716.

Tucker JA, Mintzer KA, Mullins MC. 2008. The BMP signaling gradient patterns dorsoventral tissues in a temporally progressive manner along the anteroposterior axis. Dev Cell 14: 108-119.

Turing AM. 1952. The chemical basis of morphogenesis. Philos Trans R Soc B 237: 37-72.

Umulis DM, Serpe M, O'Connor MB, Othmer HG. 2006. Robust, bistable patterning of the dorsal surface of the Drosophila embryo. Proc Natl Acad Sci 103: 11613 11618. von der Hardt S, Bakkers J, Inbal A, Carvalho L, Solnica-Krezel L, Heisenberg C, Hammerschmidt M. 2007. The Bmp gradient of the zebrafish gastrula guides migrating lateral cells by regulating cell-cell adhesion. Curr Biol 17: 475-487.

Waddington CH. 1932. Experiments on the development of chick and duck embryos, cultivated in vitro. Phil Trans $R$ Soc B 221: 179-230.

Wang X, Harris RE, Bayston LJ, Ashe HL. 2008. Type IV collagens regulate BMP signalling in Drosophila. Nature 455: $72-77$.

Wang Y, Ferguson EL. 2005. Spatial bistability of Dpp-receptor interactions during Drosophila dorsalventral patterning. Nature 434: 229-234.

Wolpert L. 1969. Positional information and the spatial pattern of cellular differentiation. J Theor Biol 25: 1-47.

Wozney JM, Rosen V, Celeste AJ, Mitsock LM, Whitters MJ, Kriz RW, Hewick RM, Wang EA. 1988. Novel regulators of bone formation: Molecular clones and activities. Science 242: 1528-1534.

Xie J, Fisher S. 2005. Twisted gastrulation enhances BMP signaling through chordin dependent and independent mechanisms. Development 132: 383-391.

Yabe T, Shimizu T, Muraoka O, Bae Y, Hirata T, Nojima H, Kawakami A, Hirano T, Hibi M. 2003. Ogon/Secreted Frizzled functions as a negative feedback regulator of Bmp signaling. Development 130: 2705-2716.

Zakin L, Metzinger CA, Chang EY, Coffinier C, Robertis ED. 2008. Development of the vertebral morphogenetic field in the mouse: Interactions between Crossveinless- 2 and Twisted Gastrulation. Dev Biol 323: 6-18.

Zeng L, Han Z, Ma W. 2005. Elucidation of subfamily segregation and intramolecular coevolution of the olfactomedin-like proteins by comprehensive phylogenetic analysis and gene expression pattern assessment. FEBS Lett 579: 5443-5453.

Zhang J, Huang Y, Qiu L, Nickel J, Sebald W. 2007a. von Willebrand factor type $\mathrm{C}$ domain-containing proteins regulate bone morphogenetic protein signaling through different recognition mechanisms. J Biol Chem 282: 20002-20014.

Zhang Y, Lander AD, Nie Q. 2007b. Computational analysis of BMP gradients in dorsal-ventral patterning of the zebrafish embryo. J Theor Biol 248: 579-589.

Zhang J, Qiu L, Kotzsch A, Weidauer S, Patterson L, Hammerschmidt M, Sebald W, Mueller TD. 2008. Crystal structure analysis reveals how the Chordin family member crossveinless 2 blocks BMP-2 receptor binding. Dev Cell 14: 739-750.

Zimmerman LB, De Jesús-Escobar JM, Harland RM. 1996. The Spemann organizer signal noggin binds and inactivates bone morphogenetic protein 4. Cell 86: 599-606. 


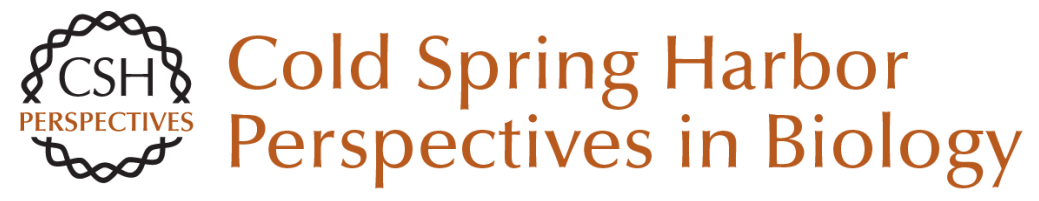

\title{
Systems Biology of the Self-regulating Morphogenetic Gradient of the Xenopus Gastrula
}

\author{
Jean-Louis Plouhinec and E. M. De Robertis
}

Cold Spring Harb Perspect Biol 2009; doi: 10.1101/cshperspect.a001701 originally published online July 29, 2009

\section{Subject Collection Generation and Interpretation of Morphogen Gradients}

Regulation of Organ Growth by Morphogen

Gradients

Gerald Schwank and Konrad Basler

Signaling Gradients during Paraxial Mesoderm

Development

Alexander Aulehla and Olivier Pourquié

Morphogen Gradient Formation

Ortrud Wartlick, Anna Kicheva and Marcos

González-Gaitán

Nodal Morphogens

Alexander F. Schier

Gradients and the Specification of Planar Polarity in the Insect Cuticle

David Strutt

Vertebrate Limb Development: Moving from Classical Morphogen Gradients to an Integrated 4-Dimensional Patterning System

Jean-Denis Bénazet and Rolf Zeller

Establishing and Interpreting Graded Sonic Hedgehog Signaling during Vertebrate Neural Tube Patterning: The Role of Negative Feedback Vanessa Ribes and James Briscoe
Gradients in Planarian Regeneration and

Homeostasis

Teresa Adell, Francesc Cebrià and Emili Saló

Shaping Morphogen Gradients by Proteoglycans Dong Yan and Xinhua Lin

Forming Patterns in Development without Morphogen Gradients: Scattered Differentiation and Sorting Out

Robert R. Kay and Christopher R.L. Thompson

Robust Generation and Decoding of Morphogen Gradients

Naama Barkai and Ben-Zion Shilo

Models for the Generation and Interpretation of Gradients

Hans Meinhardt

Graded Dorsal and Differential Gene Regulation in the Drosophila Embryo

Gregory T. Reeves and Angelike Stathopoulos

Chemical Gradients and Chemotropism in Yeast Robert A. Arkowitz

For additional articles in this collection, see http://cshperspectives.cshlp.org/cgi/collection/

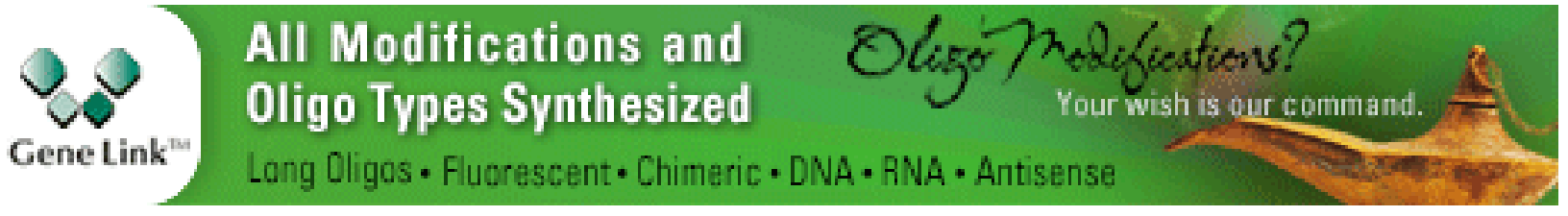


Systems Biology of the Self-regulating

Morphogenetic Gradient of the Xenopus Gastrula Jean-Louis Plouhinec and E. M. De Robertis
Gradients in the Brain: The Control of the Development of Form and Function in the Cerebral Cortex

Stephen N. Sansom and Frederick J. Livesey

For additional articles in this collection, see http://cshperspectives.cshlp.org/cgi/collection/

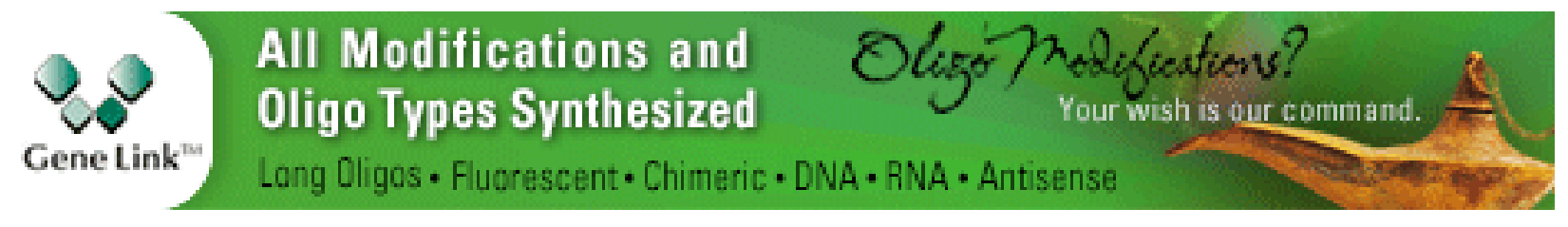

Copyright @ 2009 Cold Spring Harbor Laboratory Press; all rights reserved 